\title{
A Unified Model for Word Sense Representation and Disambiguation
}

\author{
Xinxiong Chen, Zhiyuan Liu, Maosong Sun \\ State Key Laboratory of Intelligent Technology and Systems \\ Tsinghua National Laboratory for Information Science and Technology \\ Department of Computer Science and Technology \\ Tsinghua University, Beijing 100084, China \\ cxx.thu@gmail.com, \{lzy, sms\}@tsinghua.edu.cn
}

\begin{abstract}
Most word representation methods assume that each word owns a single semantic vector. This is usually problematic because lexical ambiguity is ubiquitous, which is also the problem to be resolved by word sense disambiguation. In this paper, we present a unified model for joint word sense representation and disambiguation, which will assign distinct representation$\mathrm{s}$ for each word sense. ${ }^{1}$ The basic idea is that both word sense representation (WSR) and word sense disambiguation (WSD) will benefit from each other: (1) highquality WSR will capture rich information about words and senses, which should be helpful for WSD, and (2) high-quality WSD will provide reliable disambiguated corpora for learning better sense representations. Experimental results show that, our model improves the performance of contextual word similarity compared to existing WSR methods, outperforms stateof-the-art supervised methods on domainspecific WSD, and achieves competitive performance on coarse-grained all-words WSD.
\end{abstract}

\section{Introduction}

Word representation aims to build vectors for each word based on its context in a large corpus, usually capturing both semantic and syntactic information of words. These representations can be used as features or inputs, which are widely employed in information retrieval (Manning et al., 2008), document classification (Sebastiani, 2002) and other NLP tasks.

\footnotetext{
${ }^{1}$ Our sense representations can be downloaded at http: //pan.baidu.com/s/1eQcPK8i.
}

Most word representation methods assume each word owns a single vector. However, this is usually problematic due to the homonymy and polysemy of many words. To remedy the issue, Reisinger and Mooney (2010) proposed a multi-prototype vector space model, where the contexts of each word are first clustered into groups, and then each cluster generates a distinct prototype vector for a word by averaging over all context vectors within the cluster. Huang et al. (2012) followed this idea, but introduced continuous distributed vectors based on probabilistic neural language models for word representations.

These cluster-based models conduct unsupervised word sense induction by clustering word contexts and, thus, suffer from the following issues:

- It is usually difficult for these cluster-based models to determine the number of clusters. Huang et al. (2012) simply cluster word contexts into static $K$ clusters for each word, which is arbitrary and may introduce mistakes.

- These cluster-based models are typically offline, so they cannot be efficiently adapted to new senses, new words or new data.

- It is also troublesome to find the sense that a word prototype corresponds to; thus, these cluster-based models cannot be directly used to perform word sense disambiguation.

In reality, many large knowledge bases have been constructed with word senses available online, such as WordNet (Miller, 1995) and Wikipedia. Utilizing these knowledge bases to learn word representation and sense representation is a natural choice. In this paper, we present a unified model for both word sense representation and disambiguation based on these knowledge bases and large-scale text corpora. The unified model 
can (1) perform word sense disambiguation based on vector representations, and (2) learn continuous distributed vector representation for word and sense jointly.

The basic idea is that, the tasks of word sense representation (WSR) and word sense disambiguation (WSD) can benefit from each other: (1) high-quality WSR will capture rich semantic and syntactic information of words and senses, which should be helpful for WSD; (2) high-quality WS$\mathrm{D}$ will provide reliable disambiguated corpora for learning better sense representations.

By utilizing these knowledge bases, the problem mentioned above can be overcome:

- The number of senses of a word can be decided by the expert annotators or web users.

- When a new sense appears, our model can be easily applied to obtain a new sense representation.

- Every sense vector has a corresponding sense in these knowledge bases.

We conduct experiments to investigate the performance of our model for both WSR and WSD. We evaluate the performance of WSR using a contextual word similarity task, and results show that out model can significantly improve the correlation with human judgments compared to baselines. We further evaluate the performance on both domain-specific WSD and coarse-grained allwords WSD, and results show that our model yields performance competitive with state-of-theart supervised approaches.

\section{Methodology}

We describe our method as a 3-stage process:

1. Initializing word vectors and sense vectors. Given large amounts of text data, we first use the Skip-gram model (Mikolov et al., 2013), a neural network based language model, to learn word vectors. Then, we assign vector representations for senses based on their definitions (e.g, glosses in WordNet).

2. Performing word sense disambiguation. Given word vectors and sense vectors, we propose two simple and efficient WSD algorithms to obtain more relevant occurrences for each sense.

\section{Learning sense vectors from relevant oc-} currences. Based on the relevant occurrences of ambiguous words, we modify the training objective of Skip-gram to learn word vectors and sense vectors jointly. Then, we obtain the sense vectors directly from the model.

Before illustrating the three stages of our method in Sections 2.2, 2.3 and 2.4, we briefly introduce our sense inventory, WordNet, in Section 2.1. Note that, although our experiments will use the WordNet sense inventory, our model is not limited to this particular lexicon. Other knowledge bases containing word sense distinctions and definitions can also serve as input to our model.

\subsection{WordNet}

WordNet (Miller, 1995) is the most widely used computational lexicon of English where a concep$\mathrm{t}$ is represented as a synonym set, or synset. The words in the same synset share a common meaning. Each synset has a textual definition, or gloss. Table 1 shows the synsets and the corresponding glosses of the two common senses of bank.

Before introducing the method in detail, we introduce the notations. The unlabeled texts are denoted as $R$, and the vocabulary of the texts is denoted as $W$. For a word $w$ in $W, w_{s_{i}}$ is the $i$ th sense in WordNet $W N$. Each sense $w_{s_{i}}$ has a gloss $\operatorname{gloss}\left(w_{s_{i}}\right)$ in $W N$. The word embedding of $w$ is denoted as $\operatorname{vec}(w)$, and the sense embedding of its $i$ th sense $w_{s_{i}}$ is denoted as $\operatorname{vec}\left(w_{s_{i}}\right)$.

\subsection{Initializing Word Vectors and Sense Vectors}

Initializing word vectors. First, we use Skipgram to train the word vectors from large amounts of text data. We choose Skip-gram for its simplicity and effectiveness. The training objective of Skip-gram is to train word vector representations that are good at predicting its context in the same sentence (Mikolov et al., 2013).

More formally, given a sequence of training words $w_{1}, w_{2}, w_{3}, \ldots, w_{T}$, the objective of Skipgram is to maximize the average log probability

$$
\frac{1}{T} \sum_{t=1}^{T}\left(\sum_{-k \leq j \leq k, j \neq 0} \log p\left(w_{t+j} \mid w_{t}\right)\right)
$$

where $k$ is the size of the training window. The inner summation spans from $-k$ to $k$ to compute 


\begin{tabular}{|l|l|l|}
\hline Sense & Synset & Gloss \\
\hline bank $_{s_{1}}$ & bank & $\begin{array}{l}\text { (sloping land (especially the slope beside a body of water)) } \\
\text { "they pulled the canoe up on the bank"; } \\
\text { "he sat on the bank of the river and watched the currents" }\end{array}$ \\
\hline bank $_{s_{2}}$ & $\begin{array}{l}\text { depository institution, } \\
\text { bank, } \\
\text { banking concern, } \\
\text { banking company }\end{array}$ & $\begin{array}{l}\text { (a financial institution that accepts deposits and channels the } \\
\text { money into lending activities) } \\
\text { "he cashed a check at the bank"; } \\
\text { "that bank holds the mortgage on my home" }\end{array}$ \\
\hline
\end{tabular}

Table 1: Example of a synset in WordNet.

the log probability of correctly predicting the word $w_{t+j}$ given the word in the middle $w_{t}$. The outer summation covers all words in the training data.

The prediction task is performed via softmax, a multiclass classifier. There, we have

$$
p\left(w_{t+j} \mid w_{t}\right)=\frac{\exp \left(\operatorname{vec}^{\prime}\left(w_{t+j}\right)^{\top} \operatorname{vec}\left(w_{t}\right)\right)}{\sum_{w=1}^{W} \exp \left(\operatorname{vec}^{\prime}(w)^{\top} \operatorname{vec}\left(w_{t}\right)\right)}
$$

where $\operatorname{vec}(w)$ and $\operatorname{vec}^{\prime}(w)$ are the "input" and "output" vector representations of $w$. This formulation is impractical because the cost of computing $p\left(w_{t+j} \mid w_{t}\right)$ is proportional to $W$, which is often large $\left(10^{5}-10^{7}\right.$ terms $)$.

Initializing sense vectors. After learning the word vectors using the Skip-gram model, we initialize the sense vectors based on the glosses of senses. The basic idea of the sense vector initialization is to represent the sense by using the similar words in the gloss. From the content words in the gloss, we select those words whose cosine similarities with the original word are larger than a similarity threshold $\delta$. Formally, for each sense $w_{s_{i}}$ in $W N$, we first define a candidate set from $\operatorname{gloss}\left(w_{s_{i}}\right)$

$$
\begin{array}{r}
\operatorname{cand}\left(w_{s_{i}}\right)=\left\{u \mid u \in \operatorname{gloss}\left(w_{s_{i}}\right), u \neq w,\right. \\
\operatorname{POS}(u) \in C W, \cos (\operatorname{vec}(w), \operatorname{vec}(u))>\delta\}
\end{array}
$$

where $\operatorname{POS}(u)$ is the part-of-speech tagging of the word $u$ and $C W$ is the set of all possible part-ofspeech tags that content words could have. In this paper, $C W$ contains the following tags: noun, verb, adjective and adverb.

Then the average of the word vectors in $\operatorname{cand}\left(w_{s_{i}}\right)$ is used as the initialization value of the sense vector $\operatorname{vec}\left(w_{s_{i}}\right)$.

$$
\operatorname{vec}\left(w_{s_{i}}\right)=\frac{1}{\left|\operatorname{cand}\left(w_{s_{i}}\right)\right|} \sum_{u \in \operatorname{cand}\left(w_{s_{i}}\right)} \operatorname{vec}(u)
$$

For example, in WordNet, the gloss of the sense bank $_{s_{1}}$ is "sloping land (especially the slope beside a body of water)) they pulled the canoe up on the bank; he sat on the bank of the river and watched the currents". The gloss contains a definition of the sense and two examples of the sense. The content words and the cosine similarities with the word "bank" are listed as follows: (sloping, 0.12), (land, 0.21), (slope, 0.17), (body, 0.01), (water, 0.10), (pulled, 0.01), (canoe, 0.09), (sat, 0.06), (river, 0.43), (watch, -0.11), (currents, 0.01). If the threshold, $\delta$, is set to 0.05 , then $\operatorname{cand}\left(\mathrm{bank}_{s_{1}}\right)$ is \{sloping, land, slope, water, canoe, sat, river\}. Then the average of the word vectors in $\operatorname{cand}\left(\right.$ bank $\left._{s_{i}}\right)$ is used as the initialization value of $\operatorname{vec}\left(\right.$ bank $\left._{s_{i}}\right)$.

\subsection{Performing Word Sense Disambiguation.}

One of the state-of-the-art WSD results can be obtained using exemplar models, i.e., the word meaning is modeled by using relevant occurrences only, rather than merging all of the occurrences into a single word vector (Erk and Pado, 2010). Inspired by this idea, we perform word sense disambiguation to obtain more relevant occurrences.

Here, we perform knowledge-based word sense disambiguation for training data on an all-words setting, i.e., we will disambiguate all of the content words in a sentence. Formally, the sentence $S$ is a sequence of words $\left(w_{1}, w_{2}, \ldots, w_{n}\right)$, and we will identify a mapping $M$ from words to senses such that $M(i) \in \operatorname{Senses}_{W N}\left(w_{i}\right)$, where $\operatorname{Senses}_{W N}\left(w_{i}\right)$ is the set of senses encoded in the $W N$ for word $w_{i}$. For sentence $S$, there are $\prod_{i=1}^{n}\left|\operatorname{Sense}_{W N}\left(w_{i}\right)\right|$ possible mapping answers, which are impractical to compute. Thus, we design two simple algorithms, L2R (left to right) algorithm and S2C (simple to complex) algorithm, for word sense disambiguation based on the sense vectors.

The main difference between $\mathrm{L} 2 \mathrm{R}$ and $\mathrm{S} 2 \mathrm{C}$ is 
the order of words when performing word sense disambiguation. When given a sentence, the L2R algorithm disambiguates the words from left to right (the natural order of a sentence), whereas the $\mathrm{S} 2 \mathrm{C}$ algorithm disambiguates the words with fewer senses first. The main idea of S2C algorithm is that the words with fewer senses are easier to disambiguate, and the disambiguation result can be helpful to disambiguate the words with more senses. Both of the algorithms have three steps:

Context vector initialization. Similar to the initialization of sense vectors, we use the average of all of the content words' vectors in a sentence as the initialization vector of context.

$$
\operatorname{vec}(\text { context })=\frac{1}{|\operatorname{cand}(S)|} \sum_{u \in \operatorname{cand}(S)} \operatorname{vec}(u)
$$

where $\operatorname{cand}(S)$ is the set of content words $\operatorname{cand}(S)=\{u \mid u \in S, P O S(u) \in C W\}$.

Ranking words. For L2R, we do nothing in this step. For S2C, we rank the words based on the ascending order of $\left|\operatorname{Senses}_{W N}\left(w_{i}\right)\right|$.

Word sense disambiguation. For both $\mathrm{L} 2 \mathrm{R}$ and $\mathrm{S} 2 \mathrm{C}$, we denote the order of words as $L$ and perform word sense disambiguation according to $L$.

First, we skip a word if the word is not a content word or the word is monosemous $\left(\left|\operatorname{Senses}_{W N}\left(w_{i}\right)\right|=1\right)$. Then, for each word in $L$, we can compute the cosine similarities between the context vector and its sense vectors. We choose the sense that yields the maximum cosine similarity as its disambiguation result. If the score margin between the maximum and the second maximum is larger than the threshold $\varepsilon$, we are confident with the disambiguation result of $w_{i}$ and then use the sense vector to replace the word vector in the context vector. Thus, we obtain a more accurate context vector for other words that are still yet to be disambiguated.

For example, given a sentence "He sat on the bank of the lake", we first explain how S2C works. In the sentence, there are three content words, "sat", "bank" and "lake", to be disambiguated. First, the sum of the three word vectors is used as the initialization of the context vector. Then we rank the words by $\mid$ Senses $_{W} N\left(w_{i}\right) \mid$, in ascending order, that is, lake ( 3 senses), bank (10 senses), sat (10 senses). We first disambiguate the word "lake" based on the similarities between its sense vectors and context vector. If the score margin is larger

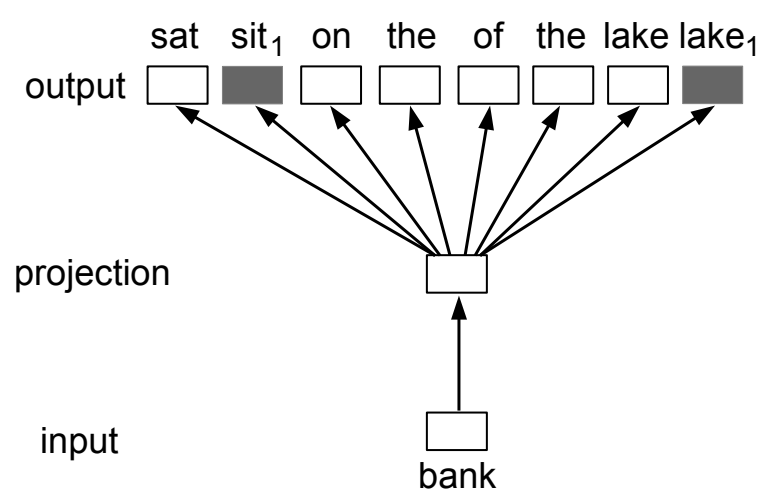

Figure 1: The architecture of our model. The training objective of Skip-gram is to train word vector representations that are not only good at predicting its context words but are also good at predicting its context words' senses. The center word "bank" is used to predict not only its context words but also the sense "sit ${ }_{1}$ " and "lake 1 ".

than the threshold $\varepsilon$, then we are confident with this disambiguation result and replace the word vector with the sense vector to update the contex$\mathrm{t}$ vector. It would be helpful to disambiguate the next word, "bank". We repeat this process until all three words are disambiguated.

For L2R, the order of words to be disambiguated will be "sat", "bank" and "lake". In this time, when disambiguating "bank" (10 senses), we still don't know the sense of "lake" (3 senses).

\subsection{Learning Sense Vectors from Relevant Occurrences.}

Based on the disambiguation result, we modify the training objective of Skip-gram and train the sense vectors directly from the large-scale corpus. Our training objective is to train the vector representations that are not only good at predicting its context words but are also good at predicting its context words' senses. The architecture of our model is shown in Figure 1.

More formally, given the disambiguation result $M\left(w_{1}\right), M\left(w_{2}\right), M\left(w_{3}\right), \ldots, M\left(w_{T}\right)$, the training objective is modified to

$$
\frac{1}{T} \sum_{t=1}^{T}\left(\sum_{j=-k}^{k} \log \left\{p\left(w_{t+j} \mid w_{t}\right) p\left(M\left(w_{t+j}\right) \mid w_{t}\right)\right\}\right)
$$

where $k$ is the size of the training window. The inner summation spans from $-k$ to $k$ to compute the log probability of correctly predicting the word $w_{t+j}$ and the log probability of correctly predicting 
the sense $M\left(w_{t+j}\right)$ given the word in the middle $w_{t}$. The outer summation covers all words in the training data.

Because not all of the disambiguation results are correct, we only disambiguate the words that we are confident in. Similar to step 3 of our WSD algorithm, we only disambiguate words under the condition that the score margin between the maximum and the second maximum is larger than the score margin threshold, $\varepsilon$.

We also use the softmax function to define $p\left(w_{t+j} \mid w_{t}\right)$ and $p\left(M\left(w_{t+j}\right) \mid w_{t}\right)$. Then, we use hierarchical softmax (Morin and Bengio, 2005) to greatly reduce the computational complexity and learn the sense vectors directly from the relevant occurrences.

\section{Experiments}

In this section, we first present the nearest neighbors of some words and their senses, showing that our sense vectors can capture the semantics of words. Then, we use three tasks to evaluate our unified model: a contextual word similarity task to evaluate our sense representations, and two standard WSD tasks to evaluate our knowledge-based WSD algorithm based on the sense vectors. Experimental results show that our model not only improves the correlation with human judgments on the contextual word similarity task but also outperforms state-of-the-art supervised WSD system$s$ on domain-specific datasets and competes with them in a coarse-grained all-words setting.

We choose Wikipedia as the corpus to train the word vectors because of its wide coverage of topics and words usages. We use an English Wikipedia database dump from October $2013^{2}$, which includes roughly 3 million articles and 1 billion tokens. We use Wikipedia Extractor ${ }^{3}$ to preprocess the Wikipedia pages and only save the content of the articles.

We use word2 $\mathrm{vec}^{4}$ to train Skip-gram. We use the default parameters of word $2 \mathrm{vec}$ and the dimension of the vector representations is 200 .

We use WordNet ${ }^{5}$ as our sense inventory. The datasets for different tasks are tagged with different versions of WordNet. The version of WordNet

\footnotetext{
${ }^{2}$ http: // download.wikipedia.org.

${ }^{3}$ The tool is available from http://medialab.di. unipi.it/wiki/Wikipedia_Extractor.

${ }^{4}$ The code is available from https://code. google.com/p/word2vec/.

5 http: //wordnet.princeton. edu/.
}

\begin{tabular}{|c|l|}
\hline Word or sense & Nearest neighbors \\
\hline bank & banks, IDBI, CitiBank \\
\hline bank $_{s_{1}}$ & river, slope, Sooes \\
\hline bank $_{s_{2}}$ & mortgage, lending, loans \\
\hline star & stars, stellar, trek \\
\hline star $_{s_{1}}$ & $\begin{array}{l}\text { photosphere, radiation, } \\
\text { gamma-rays }\end{array}$ \\
\hline star $_{s_{2}}$ & someone, skilled, genuinely \\
\hline plant $^{\text {plant }} s_{s_{1}}$ & $\begin{array}{l}\text { flants, glavaticevo, herbaceous } \\
\text { manufacturing }\end{array}$ \\
\hline plant $_{s_{2}}$ & $\begin{array}{l}\text { locomotion, organism, } \\
\text { organisms }\end{array}$ \\
\hline
\end{tabular}

Table 2: Nearest neighbors of word vectors and sense vectors learned by our model based on cosine similarity. The subscript of each sense label corresponds to the index of the sense in WordNet. For example, bank $s_{s_{2}}$ is the second sense of the word bank in WordNet.

is 1.7 for the domain-specific WSD task and 2.1 for the coarse-grained WSD task.

We use the S2C algorithm described in Section 2.3 to perform word sense disambiguation to obtain more relevant occurrences for each sense. We compare S2C and L2R on the coarse-grained WS$\mathrm{D}$ task in a all-words setting.

The experimental results of our model are obtained by setting the similarity threshold as $\delta=0$ and the score margin threshold as $\varepsilon=0.1$. The influence of parameters on our model can be found in Section 3.5.

\subsection{Examples for Sense Vectors}

Table 2 shows the nearest neighbors of word vectors and sense vectors based on cosine similarity. We see that our sense representations can identify different meanings of a word, allowing our model to capture more semantic and syntactic relationships between words and senses. Note that each sense vector in our model corresponds to a sense in WordNet; thus, our sense vectors can be used to perform knowledge-based word sense disambiguation, whereas the vectors of cluster-based models cannot.

\subsection{Contextual Word Similarity}

Experimental setting. A standard dataset for evaluating a vector-space model is the WordSim353 dataset (Finkelstein et al., 2001), which con- 


\begin{tabular}{|l|r|}
\hline Model & $\rho \times 100$ \\
\hline C\&W-S & 57.0 \\
\hline Huang-S & 58.6 \\
\hline Huang-M AvgSim & 62.8 \\
\hline Huang-M AvgSimC & 65.7 \\
\hline Our Model-S & 64.2 \\
\hline Our Model-M AvgSim & 66.2 \\
\hline Our Model-M AvgSimC & $\mathbf{6 8 . 9}$ \\
\hline
\end{tabular}

Table 3: Spearman's $\rho$ on the SCWS dataset. Our Model-S uses one representation per word to compute similarities, while Our Model-M uses one representation per sense to compute similarities. AvgSim calculates the similarity with each sense contributing equally, while AvgSimC weighs the sense according to the probability of the word choosing that sense in context $c$.

sists of 353 pairs of nouns. However, each pair of nouns in WordSim-353 is presented without context. This is problematic because the meanings of homonymous and polysemous words depend highly on the words' contexts. Thus we choose the Stanford's Contextual Word Similarities (SCWS) dataset from (Huang et al., 2012) ${ }^{6}$. The SCWS dataset contains 2003 pairs of words and each pair is associated with 10 human judgments on similarity on a scale from 0 to 10 . In the SCWS dataset, each word in a pair has a sentential context.

In our experiments, the similarity between a pair of words $\left(w, w^{\prime}\right)$ is computed as follows:

$$
\begin{aligned}
& \operatorname{Avg} \operatorname{Sim} C\left(w, w^{\prime}\right)=\frac{1}{M N} \sum_{i=1}^{M} \sum_{j=1}^{N} \\
& \quad p(i \mid w, c) p\left(j \mid w^{\prime}, c^{\prime}\right) d\left(\operatorname{vec}\left(w_{s_{i}}\right), \operatorname{vec}\left(w_{s_{j}}^{\prime}\right)\right)
\end{aligned}
$$

where $p(i \mid w, c)$ is the likelihood that word $w$ chooses its $i$ th sense given context $c$. $d\left(v e c, v e c^{\prime}\right)$ is a function computing the similarity between two vectors, and here we use cosine similarity.

Results and discussion. For evaluation, we compute the Spearman correlation between a model's computed similarity scores and human judgements. Table 3 shows our results compared to previous methods, including (Collobert and Weston, 2008)'s language model (C\&W), and Huang's model which utilize the global context and multi-prototype to improve the word representations.

\footnotetext{
${ }^{6}$ The dataset can be downloaded at http://ai. stanford.edu/ ehhuang/.
}

From Table 3, we observe that:

- Our single-vector version outperforms Huang's single-vector version. This indicates that, by training the word vectors and sense vectors jointly, our model can better capture the semantic relationships between words and senses.

- With one representation per sense, our model can outperform the single-vector version without using context (66.2 vs. 64.2).

- Our model obtains the best performance (68.9) by using AvgSimC, which takes context into account.

\subsection{Domain-Specific WSD}

Experimental setting. We use Wikipedia as training data because of its wide coverage for specific domains. To test our performance on domain word sense disambiguation, we evaluated our system on the dataset published in (Koeling et al., 2005). This dataset consists of examples retrieved from the Sports and Finance sections of the Reuters corpus. 41 words related to the Sports and Finance domains were selected, with an average polysemy of 6.7 senses, ranging from 2 to 13 senses.

Approximately 100 examples for each word were annotated with senses from WordNet v.1.7 by three reviewers, yielding an inter-tagger agreement of 65\%. (Koeling et al., 2005) did not clarify how to select the "correct" sense for each word, so we followed the work of (Agirre et al., 2009) and, used the sense chosen by the majority of taggers as the correct answer.

Baseline methods. As a baseline, we use the most frequent WordNet sense (MFS), as well as a random sense assignment. We also compare our results with four systems ${ }^{7}$ : Static PageRank (Agirre et al., 2009), the $k$ nearest neighbor algorith$\mathrm{m}(k-\mathrm{NN})$, Degree (Navigli and Lapata, 2010) and Personalized PageRank (Agirre et al., 2009).

Static PageRank applies traditional PageRank over the semantic graph based on WordNet and obtains a context-independent ranking of word senses.

$k$-NN is a widely used classification method, where neighbors are the $k$ labeled examples most

\footnotetext{
${ }^{7}$ We compare only with those systems performing tokenbased WSD, i.e., disambiguating each instance of a target word separately.
} 


\begin{tabular}{|c|c|c|}
\hline Algorithm & $\begin{array}{c}\text { Sports } \\
\text { Recall }\end{array}$ & $\begin{array}{c}\text { Finance } \\
\text { Recall }\end{array}$ \\
\hline Random BL & 19.5 & 19.6 \\
MFS BL & 19.6 & 37.1 \\
\hline k-NN & 30.3 & 43.4 \\
Static PR & 20.1 & 39.6 \\
Personalized PR & 35.6 & 46.9 \\
Degree & 42.0 & 47.8 \\
\hline Our Model & $\mathbf{5 7 . 3}$ & $\mathbf{6 0 . 6}$ \\
\hline
\end{tabular}

Table 4: Performance on the Sports and Finance sections of the dataset from (Koeling et al., 2005).

similar to the test example. The $k$-NN system is trained on SemCor (Miller et al., 1993), the largest publicly available annotated corpus.

Degree and Personalized PageRank are stateof-the-art systems that exploit WordNet to build a semantic graph and exploit the structural properties of the graph in order to choose the appropriate senses of words in context.

Results and discussion. Similar to other work on this dataset, we use recall (the ratio of correct sense labels to the total labels in the gold standard) as our evaluation measure. Table 4 shows the results of different WSD systems on the dataset, and the best results are shown in bold. The differences between other results and the best result in each column of the table are statistically significant at $p<0.05$.

The results show that:

- Our model outperforms $k-\mathrm{NN}$ on the two domains by a large margin, supporting the findings from (Agirre et al., 2009) that knowledge-based systems perform better than supervised systems when evaluated across different domains.

- Our model also achieves better results than the state-of-the-art system $(+15.3 \%$ recall on Sports and $+12.8 \%$ recall on Finance against Degree). The reason for this is that when dealing with short sentences or context words that are not in WordNet, our model can still compute similarity based on the context vector and sense vectors, whereas Degree will have difficulty building the semantic graph.

- Moreover, our model achieves the best performance by only using the unlabeled text data and the definitions of senses, whereas other

\begin{tabular}{|c|c|c|c|}
\hline Algorithm & Type & $\begin{array}{c}\text { Nouns only } \\
F_{1}\end{array}$ & $\begin{array}{c}\text { All words } \\
F_{1}\end{array}$ \\
\hline Random BL & U & 63.5 & 62.7 \\
MFS BL & Semi & 77.4 & 78.9 \\
\hline SUSSX-FR & Semi & 81.1 & 77.0 \\
NUS-PT & S & 82.3 & $\mathbf{8 2 . 5}$ \\
SSI & Semi & $\mathbf{8 4 . 1}$ & $\mathbf{8 3 . 2}$ \\
Degree $^{\text {Semi }}$ & $\mathbf{8 5 . 5}$ & $\mathbf{8 1 . 7}$ \\
\hline Our Model $_{L 2 R}$ & U & 79.2 & 73.9 \\
Our Model $_{S 2 C}$ & U & 81.6 & 75.8 \\
Our Model $_{L 2 R}$ & Semi & 82.5 & 79.6 \\
Our Model $_{S 2 C}$ & Semi & $\mathbf{8 5 . 3}$ & $\mathbf{8 2 . 6}$ \\
\hline
\end{tabular}

Table 5: Performance on Semeval-2007 coarsegrained all-words WSD. In the type column, $\mathrm{U}$, Semi and $\mathrm{S}$ stand for unsupervised, semisupervised and supervised, respectively. The differences between the results in bold in each column of the table are not statistically significant at $p<0.05$.

methods rely greatly on high-quality semantic relations or annotated data, which are hard to acquire.

\subsection{Coarse-grained WSD}

Experimental setting. We also evaluate our WSD model on the Semeval-2007 coarse-grained all-words WSD task (Navigli et al., 2007). There are multiple reasons that we perform experiments in a coarse-grained setting: first, it has been argued that the fine granularity of WordNet is one of the main obstacles to accurate WSD (cf. the discussion in (Navigli, 2009)); second, the training corpus of word representations is Wikipedia, which is quite different from WordNet.

Baseline methods. We compare our model with the best unsupervised system SUSSX-FR (Koeling and McCarthy, 2007), and the best supervised system, NUS-PT (Chan et al., 2007), participating in the Semeval-2007 coarse-grained all-words task. We also compare with SSI (Navigli and Velardi, 2005) and the state-of-the-art system Degree (Navigli and Lapata, 2010). We use different baseline methods for the two WSD tasks because we want to compare our model with the stateof-the-art systems that are applicable to different datasets and show that our WSD method can perform robustly in these different WSD tasks. 
Results and discussion. We report our results in terms of $\mathrm{F}_{1}$-measure on the Semeval-2007 coarsegrained all-words dataset (Navigli et al., 2007). Table 5 reports the results for nouns (1,108 words) and all words (2,269 words). The difference between unsupervised and semi-supervised methods is whether the method uses MFS as a back-off strategy.

We can see that the $\mathrm{S} 2 \mathrm{C}$ algorithm outperforms the L2R algorithm no matter on the nouns subset or on the entire set. This indicates that words with fewer senses are easier to disambiguate, and it can be helpful to disambiguate the words with more senses.

On the nouns subset, our model yields comparable performance to SSI and Degree, and it outperforms NUS-PT and SUSSX-FR. Moreover, our unsupervised WSD method (S2C) beats the MF$\mathrm{S}$ baseline, which is notably a difficult competitor for knowledge-based systems.

On the entire set, our semi-supervised model is significantly better than SUSSX-FR, and it is comparable with SSI and Degree. In contrast to SSI, our model is simple and does not rely on a costly annotation effort to engineer the set of semantic relations.

Overall, our model achieves state-of-the-art performance on the Semeval-2007 coarse-grained allwords dataset compared to other systems, with a simple WSD algorithm that only relies on a largescale unlabeled text corpora and a sense inventory.

\subsection{Parameter Influence}

We investigate the influence of parameters on our model with coarse-grained all-words WSD task. The parameters include the similarity threshold, $\delta$, and the score margin threshold, $\varepsilon$.

Similarity threshold. In Table 6, we show the performance of domain WSD when the similarity threshold $\delta$ ranges from -0.1 to 0.3 . The cosine similarity interval is $[-1,1]$, and we focus on the performance in the interval [-0.1, 0.3] for two reasons: first, no words are removed from glosses when $\delta<-0.1$; second, nearly half of the word$\mathrm{s}$ are removed when $\delta>0.3$ and the performance drops significantly for the WSD task. From table 6 , we can see that our model achieves the best performance when $\delta=0.0$.

Score margin threshold. In Table 7, we show the performance on the coarse-grained all-words

\begin{tabular}{|l|c|c|}
\hline Parameter & Nouns only & All words \\
\hline $\boldsymbol{\delta}=-0.10$ & 79.8 & 74.3 \\
$\boldsymbol{\delta}=-0.05$ & 81.0 & 74.6 \\
$\boldsymbol{\delta}=0.00$ & $\mathbf{8 1 . 6}$ & $\mathbf{7 5 . 8}$ \\
$\boldsymbol{\delta}=0.05$ & 81.3 & 75.4 \\
$\boldsymbol{\delta}=0.10$ & 80.8 & 75.2 \\
$\boldsymbol{\delta}=0.15$ & 80.0 & 75.0 \\
$\boldsymbol{\delta}=0.20$ & 77.1 & 73.3 \\
$\boldsymbol{\delta}=0.30$ & 75.0 & 72.1 \\
\hline
\end{tabular}

Table 6: Evaluation results on the coarse-grained all-words WSD when the similarity threshold $\delta$ ranges from -0.1 to 0.3 .

\begin{tabular}{|l|c|c|}
\hline Parameter & Nouns only & All words \\
\hline$\varepsilon=0.00$ & 78.2 & 72.9 \\
$\varepsilon=0.05$ & 79.5 & 74.5 \\
$\varepsilon=0.10$ & $\mathbf{8 1 . 6}$ & $\mathbf{7 5 . 8}$ \\
$\varepsilon=0.15$ & 81.2 & 74.7 \\
$\varepsilon=0.20$ & 80.9 & 75.1 \\
$\varepsilon=0.25$ & 80.2 & 74.8 \\
$\varepsilon=0.30$ & 80.4 & 74.9 \\
\hline
\end{tabular}

Table 7: Evaluation results on the coarse-grained all-words WSD when the score margin threshold $\varepsilon$ ranges from 0.0 to 0.3 .

WSD when the score margin threshold $\varepsilon$ ranges from 0.0 to 0.3 . When $\varepsilon=0.0$, we use every disambiguation result to update the context vector. When $\varepsilon \neq 0$, we only use the confident disambiguation results to update the context vector if the score margin is larger than $\varepsilon$. Our model achieves the best performance when $\varepsilon=0.1$.

\section{Related Work}

\subsection{Word Representations}

Distributed representations for words were proposed in (Rumelhart et al., 1986) and have been successfully used in language models (Bengio et al., 2006; Mnih and Hinton, 2008) and many natural language processing tasks, such as word representation learning (Mikolov, 2012), named entity recognition (Turian et al., 2010), disambiguation (Collobert et al., 2011), parsing and tagging (Socher et al., 2011; Socher et al., 2013). They are very useful in NLP tasks because they can be used as inputs to learning algorithms or as extra word features in NLP systems. Hence, many NLP applications, such as keyword extraction (Li- 
u et al., 2010; Liu et al., 2011b; Liu et al., 2012), social tag suggestion (Liu et al., 2011a) and text classification (Baker and McCallum, 1998), may also potentially benefit from distributed word representation. The main advantage is that the representations of similar words are close in vector space, which makes generalization to novel patterns easier and model estimation more robust.

Word representations are hard to train due to the computational complexity. Recently, (Mikolov et al., 2013) proposed two particular models, Skipgram and CBOW, to learn word representations in large amounts of text data. The training objective of the CBOW model is to combine the representations of the surrounding words to predict the word in the middle, while the Skip-gram model's is to learn word representations that are good at predicting its context in the same sentence (Mikolov et al., 2013). Our paper uses the model architecture of Skip-gram.

Most of the previous vector-space models use one representation per word. This is problematic because many words have multiple senses. The multi-prototype approach has been widely studied. (Reisinger and Mooney, 2010) proposed the multi-prototype vector-space model. (Huang et al., 2012) used the multi-prototype models to learn the vector for different senses of a word. All of these models use the clustering of contexts as a word sense and can not be directly used in word sense disambiguation.

After our paper was submitted, we perceive the following recent advances: (Tian et al., 2014) proposed a probabilistic model for multi-prototype word representation. (Guo et al., 2014) explored bilingual resources to learn sense-specific word representation. (Neelakantan et al., 2014) proposed an efficient non-parametric model for multiprototype word representation.

\subsection{Knowledge-based WSD}

The objective of word sense disambiguation (WSD) is to computationally identify the meaning of words in context (Navigli, 2009). There are two approaches of WSD that assign meaning of words from a fixed sense inventory, supervised and knowledge-based methods. Supervised approaches require large labeled training sets, which are time consuming to create. In this paper, we only focus on knowledge-based word sense disambiguation.
Knowledge-based approaches exploit knowledge resources (such as dictionaries, thesauri, ontologies, collocations, etc.) to determine the senses of words in context. However, it has been shown in (Cuadros and Rigau, 2006) that the amount of lexical and semantic information contained in such resources is typically insufficient for high-performance WSD. Much work has been presented to automatically extend existing resources, including automatically linking Wikipedia to WordNet to include full use of the first WordNet sense heuristic (Suchanek et al., 2008), a graph-based mapping of Wikipedia categories to WordNet synsets (Ponzetto and Navigli, 2009), and automatically mapping Wikipedia pages to WordNet synsets (Ponzetto and Navigli, 2010).

It was recently shown that word representations can capture semantic and syntactic information between words (Mikolov et al., 2013). Some researchers tried to incorporate WordNet senses in a neural model to learn better word representations (Bordes et al., 2011). In this paper, we have proposed a unified method for word sense representation and disambiguation to extend the information contained in the vector representations to the existing resources. Our method only requires a large amount of unlabeled text to train sense representations and a dictionary to provide the definitions of word meanings, which makes it easily applicable to other resources.

\section{Conclusion}

In this paper, we present a unified model for word sense representation and disambiguation that uses one representation per sense. Experimental results show that our model improves the performance of contextual word similarity compared to existing WSR methods, outperforms state-of-theart supervised methods on domain-specific WSD, and achieves competitive performance on coarsegrained all-words WSD. Our model only requires large-scale unlabeled text corpora and a sense inventory for WSD, thus it can be easily applied to other corpora and tasks.

There are still several open problems that should be investigated further:

1. Because the senses of words change over time (new senses appear), we will incorporate cluster-based methods in our model to find senses that are not in the sense inventory. 
2. We can explore other WSD methods based on sense vectors to improve our performance. For example, (Li et al., 2010) used LDA to perform data-driven WSD in a manner similar to our model. We may integrate the advantages of these models and our model together to build a more powerful WSD system.

3. To learn better sense vectors, we can exploit the semantic relations (such as the hypernym and hyponym relations defined in WordNet) between senses in our model.

\section{Acknowledgments}

This work is supported by National Key Basic Research Program of China (973 Program 2014CB340500) and National Natural Science Foundation of China (NSFC 61133012).

\section{References}

Eneko Agirre, Oier Lopez De Lacalle, Aitor Soroa, and Informatika Fakultatea. 2009. Knowledge-based wsd and specific domains: Performing better than generic supervised wsd. In Proceedings of IJCAI, pages $1501-1506$.

L Douglas Baker and Andrew Kachites McCallum. 1998. Distributional clustering of words for text classification. In Proceedings of SIGIR, pages 96103.

Yoshua Bengio, Holger Schwenk, Jean-Sébastien Senécal, Fréderic Morin, and Jean-Luc Gauvain. 2006. Neural probabilistic language models. In Innovations in Machine Learning, pages 137-186.

Antoine Bordes, Jason Weston, Ronan Collobert, Yoshua Bengio, et al. 2011. Learning structured embeddings of knowledge bases. In Proceedings of $A A A I$, pages 301-306.

Yee Seng Chan, Hwee Tou Ng, and Zhi Zhong. 2007. Nus-pt: exploiting parallel texts for word sense disambiguation in the english all-words tasks. In Proceedings of SemEval, pages 253-256.

Ronan Collobert and Jason Weston. 2008. A unified architecture for natural language processing: Deep neural networks with multitask learning. In Proceedings of ICML, pages 160-167.

Ronan Collobert, Jason Weston, Léon Bottou, Michael Karlen, Koray Kavukcuoglu, and Pavel Kuksa. 2011. Natural language processing (almost) from scratch. JMLR, 12:2493-2537.

Montse Cuadros and German Rigau. 2006. Quality assessment of large scale knowledge resources. In Proceedings of EMNLP, pages 534-541.
Katrin Erk and Sebastian Pado. 2010. Exemplar-based models for word meaning in context. In Proceedings of ACL, pages 92-97.

Lev Finkelstein, Evgeniy Gabrilovich, Yossi Matias, Ehud Rivlin, Zach Solan, Gadi Wolfman, and Eytan Ruppin. 2001. Placing search in context: The concept revisited. In Proceedings of $W W W$, pages 406414.

Jiang Guo, Wanxiang Che, Haifeng Wang, and Ting Liu. 2014. Learning sense-specific word embeddings by exploiting bilingual resources. In Proceedings of COLING, pages 497-507.

Eric H Huang, Richard Socher, Christopher D Manning, and Andrew Y Ng. 2012. Improving word representations via global context and multiple word prototypes. In Proceedings of ACL, pages 873-882.

Rob Koeling and Diana McCarthy. 2007. Sussx: Ws$\mathrm{d}$ using automatically acquired predominant senses. In Proceedings of SemEval, pages 314-317.

Rob Koeling, Diana McCarthy, and John Carroll. 2005. Domain-specific sense distributions and predominant sense acquisition. In Proceedings of HLTEMNLP, pages 419-426.

Linlin Li, Benjamin Roth, and Caroline Sporleder. 2010. Topic models for word sense disambiguation and token-based idiom detection. In Proceedings of $A C L$, pages $1138-1147$.

Zhiyuan Liu, Wenyi Huang, Yabin Zheng, and Maosong Sun. 2010. Automatic keyphrase extraction via topic decomposition. In Proceedings of EMNLP, pages 366-376.

Zhiyuan Liu, Xinxiong Chen, and Maosong Sun. 2011a. A simple word trigger method for social tag suggestion. In Proceedings of EMNLP, pages 15771588.

Zhiyuan Liu, Xinxiong Chen, Yabin Zheng, and Maosong Sun. 2011b. Automatic keyphrase extraction by bridging vocabulary gap. In Proceedings of CoNLL, pages 135-144.

Zhiyuan Liu, Xinxiong Chen, and Maosong Sun. 2012. Mining the interests of chinese microbloggers via keyword extraction. Frontiers of Computer Science, 6(1):76-87.

Christopher D Manning, Prabhakar Raghavan, and Hinrich Schütze. 2008. Introduction to information retrieval. Cambridge University Press Cambridge.

Tomas Mikolov, Kai Chen, Greg Corrado, and Jeffrey Dean. 2013. Efficient estimation of word representations in vector space. Proceedings of ICLR.

Tomas Mikolov. 2012. Statistical Language Model$s$ Based on Neural Networks. Ph.D. thesis, Ph. D. thesis, Brno University of Technology. 
George A Miller, Claudia Leacock, Randee Tengi, and Ross T Bunker. 1993. A semantic concordance. In Proceedings of the workshop on HLT, pages 303308.

George A Miller. 1995. Wordnet: a lexical database for english. Communications of the ACM, 38(11):39-41.

Andriy Mnih and Geoffrey E Hinton. 2008. A scalable hierarchical distributed language model. In Proceedings of NIPS, pages 1081-1088.

Frederic Morin and Yoshua Bengio. 2005. Hierarchical probabilistic neural network language model. In Proceedings of the international workshop on artificial intelligence and statistics, pages 246-252.

Roberto Navigli and Mirella Lapata. 2010. An experimental study of graph connectivity for unsupervised word sense disambiguation. IEEE PAMI, 32(4):678-692.

Roberto Navigli and Paola Velardi. 2005. Structural semantic interconnections: a knowledge-based approach to word sense disambiguation. IEEE PAMI, 27(7):1075-1086.

Roberto Navigli, Kenneth C Litkowski, and Orin Hargraves. 2007. Semeval-2007 task 07: Coarsegrained english all-words task. In Proceedings of SemEval, pages 30-35.

Roberto Navigli. 2009. Word sense disambiguation: A survey. CSUR, 41(2):10.

Arvind Neelakantan, Jeevan Shankar, Alexandre Passos, and Andrew McCallum. 2014. Efficient nonparametric estimation of multiple embeddings per word in vector space. In Proceedings of EMNLP.

Simone Paolo Ponzetto and Roberto Navigli. 2009. Large-scale taxonomy mapping for restructuring and integrating wikipedia. In Proceedings of IJCAI, volume 9, pages 2083-2088.

Simone Paolo Ponzetto and Roberto Navigli. 2010. Knowledge-rich word sense disambiguation rivaling supervised systems. In Proceedings of $A C L$, pages 1522-1531.

Joseph Reisinger and Raymond J Mooney. 2010. Multi-prototype vector-space models of word meaning. In Proceedings of HLT-NAACL, pages 109 117.

David E Rumelhart, Geoffrey E Hintont, and Ronald J Williams. 1986. Learning representations by backpropagating errors. Nature, 323(6088):533-536.

Fabrizio Sebastiani. 2002. Machine learning in automated text categorization. CSUR, 34(1):1-47.

Richard Socher, Cliff C Lin, Andrew Ng, and Chris Manning. 2011. Parsing natural scenes and natural language with recursive neural networks. In Proceedings of ICML, pages 129-136.
Richard Socher, John Bauer, Christopher D Manning, and Andrew Y Ng. 2013. Parsing with compositional vector grammars. In Proceedings of ACL.

Fabian M Suchanek, Gjergji Kasneci, and Gerhard Weikum. 2008. Yago: A large ontology from wikipedia and wordnet. Web Semantics: Science, Services and Agents on the World Wide Web, 6(3):203-217.

Fei Tian, Hanjun Dai, Jiang Bian, Bin Gao, Rui Zhang, Enhong Chen, and Tie-Yan Liu. 2014. A probabilistic model for learning multi-prototype word embeddings. In Proceedings of COLING, pages 151-160.

Joseph Turian, Lev Ratinov, and Yoshua Bengio. 2010. Word representations: a simple and general method for semi-supervised learning. In Proceedings of ACL, pages 384-394. 